Please do not remove this page

RMIT

UNIVERSITY

\title{
RF-photonic link using a resonantly-enhanced Mach-Zehnder optical modulator
}

Nguyen, Giang; Visagathilagar, Yuvaraja; Mitchell, Arnan

https://researchrepository.rmit.edu.au/esploro/outputs/9921858624601341/filesAndLinks?institution=61RMIT_INST\&index=null

Nguyen, G., Visagathilagar, Y., \& Mitchell, A. (2005). RF-photonic link using a resonantly-enhanced Mach-Zehnder optical modulator. Proceedings of the IEEE International Topical Meeting on Microwave Photonics, 253-256.

https://researchrepository.rmit.edu.au/discovery/fulldisplay/alma9921858624601341/61RMIT_INST:Resea rchRepository

Repository homepage: https://researchrepository.rmit.edu.au

(c) 2005 IEEE. Personal use of this material is permitted. However, permission to reprint/republish this material for advertising or promotional purposes or for creating new collective works for resale or redistribution to servers or lists, or to reuse any copyrighted component of this work in other works must be obtained from the IEEE.

Downloaded On 2023/04/26 23:28:47 +1000 


\title{
RF-photonic Link Using A Resonantly-Enhanced Mach-Zehnder Optical Modulator
}

\author{
Thach G. Nguyen, Yuvaraja S. Visagathilagar, and Arnan Mitchell \\ School of Electrical and Computer Engineering, RMIT University, GPO Box 2476V, \\ Melbourne, Victoria 3001, Australia
}

\begin{abstract}
An RF-photonic link using an optimized resonantly-enhanced optical intensity modulator (REMZM) is demonstrated. The enhanced modulator provides a 9dB link gain improvement when compared to a commercial, broadband modulator with a 3dB bandwidth of $11 \%$. The noise figure of the link is experimentally measured as a function of optical power. Comparison to an un-enhanced modulator demonstrates that when the link noise is laser RIN limited, the enhanced modulator provides a significant improvement to noise figure. The intermodulation distortion of RE-MZM link is also analyzed and it is shown that the resonant enhancement does not impact modulation dynamic range.
\end{abstract}

Index Terms-Intergrated optics, noise, nonlinearities, optical communication, optical modulation

\section{INTRODUCTION}

Mach-Zehnder optical modulator (MZM) on $\mathrm{LiNbO}_{3}$ has been widely used in optical communication systems. Conventional modulators which employ a travelling-wave electrode structure offer very large bandwidth but low modulation efficiency. Therefore, the RF links that use MZMs suffer large link loss. In band-operation applications such as radio-on-fiber [1] and local oscillator distribution for photonic mixing [2], only a narrow bandwidth around the center frequency is required. Hence, the resonant electrode structures [3], [4] can be employed in Mach-Zehnder modulator to improve the modulation efficiency at the expense of modulation bandwidth.

To achieve good resonant enhancement, the attenuation of the modulator electrode needs to be very small so that the electrode can be made longer to increase the modulator active length. The modulation efficiency can also be improved by reducing the inherent switching voltage of the modulator. Thus, it is desireable to have an electrode structure that provide both low electrode loss and low inherent switching voltage. In [5], we have theoretically investigated the resonantly-enhanced modulator on X-cut $\mathrm{LiNbO}_{3}$ using numerical optimization. It was shown that by optimizing the electrode cross-sectional parameters, low switching voltage can be achieved with low electrode loss. The optimized modulator has been realized and it shows a significant improvement in the modulation efficiency.

In this paper, the performance of the RF-photonic link using the realized resonantly-enhanced modulator will be analyzed. The link frequency response, noise figure and dynamic range will be presented and compared with the link using the un-enhanced modulator.

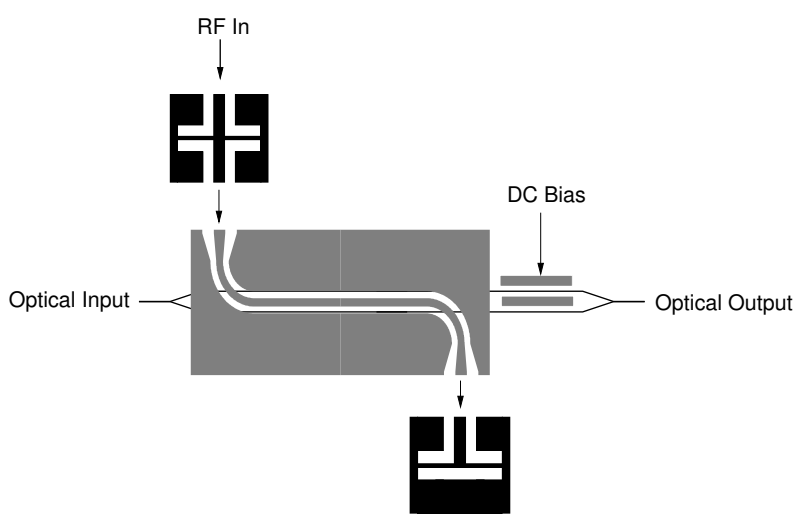

Fig. 1. Resonantly-enhanced modulator structure

\section{RESONANTLY-ENHANCED MODUlATOR AND RF-PHOTONIC LINK}

Fig. 1 illustrates the configuration of the resonantlyenhanced modulator. By placing reactive terminations on both input and output ports of the modulator electrode to form a Fabry-Perot type cavity [6], most of the microwave signal undergoes multiple reflections inside the cavity to form resonant standing waves. This process results in an enhanced electric field inside the modulator electrode, provided that low loss modulator electrode can be achieved.

With applied voltage $V_{\text {in }}$ at the input of the modulator the total accumulated phase change in two arms of a modulator due to Pockel's effect is given by:

$$
\Delta \Phi=\frac{\pi}{V_{\pi}(D C) l} \int_{0}^{l} V(z) e^{-j(2 \pi f / c) n_{o} z} d z
$$

where $V_{\pi}(D C)$ is the switching voltage of the modulator at DC, $l$ is the length of the active section of the modulator electrode, $V(z)$ is the complex voltage at point $\mathrm{z}$ along the active section of the electrode, $n_{o}$ is the effective index of the guided optical mode, and $c$ is the speed of light in free space.

The frequency dependent switching voltage is defined as:

$$
V_{\pi}(f)=\frac{\pi V_{i n}}{\Delta \Phi} .
$$

For broadband travelling wave electrode type modulator with impedance match, the voltage inside the active section $V(z)$ is smaller than the input voltage $V_{\text {in }}$ due to electrode loss. Hence, the switching voltage $V_{\pi}(f)$ at 
the frequency $f$ is always greater than the DC switching voltage $V_{\pi}(D C)$. In the case of resonantly-enhanced electrode structure, the voltage inside the active section $V_{z}$ can be greater than the input voltage $V_{i n}$. Thus, the switching voltage $V_{\pi}(f)$ at the resonant frequency can be smaller than the DC switching voltage $V_{\pi}(D C)$.

When the resonantly-enhanced modulator is used in external modulation, direct detection RF-photonic link and under small signal operation at quadrature bias, the link electrical gain is given by:

$$
\begin{aligned}
G(f)= & \left(P_{o} \alpha_{m} \alpha_{o p t} \Re \frac{\pi}{2 V_{\pi}(f)}\right)^{2} \times \\
& |1+\Gamma|^{2}\left(\frac{50 R_{D}}{50+R_{D}}\right)^{2}
\end{aligned}
$$

where $P_{o}$ is the input optical power, $\alpha_{m}$ and $\alpha_{o p t}$ are the insertion loss of the modulator and optical fiber respectively, $\Re$ is the responsivity of the photodetector, $\Gamma=\left(Z_{m}-50\right) /\left(Z_{m}+50\right)$ is the voltage reflection coefficient at the modulator input, $Z_{m}$ is the modulator impedance, and $R_{D}$ is the termination resistance of the photodetector. The link is assumed to be terminated at $50 \Omega$ input and output. It is clear that the link gain can be improved by using a modulator with low switching voltage and high optical power.

\section{EXPERIMENTAL RESUlTS}

In [5], the optimal electrode configuration to maximizing resonantly-enhanced modulator efficiency has been identified. Here it was found that optimal performance is achieved by minimizing electrode loss, even at the expense of inherent modulator efficiency. The modulator was fabricated in $\mathrm{X}$-cut $\mathrm{LiNbO}_{3}$. The fabricated modulator shows a DC switching voltage of $2.5 \mathrm{~V}$. The optical insertion loss of the modulator was measured to be of $13 \mathrm{~dB}$. This excessive loss is due to the defects in the end-face polishing of this particular modulator. The typical insertion loss of fabricated modulators is about $5 \mathrm{~dB}$. The reactive terminations were realized by shorted CPW stub circuits on ceramic substrate. The terminations were then wire-bonded to the modulator tapers to form the resonantly-enhanced modulator. In all experiments the modulator was biased at quadrature for linear operation.

Fig. 2 shows the frequency dependence of the link gain of the RF-photonic links formed using the resonantlyenhanced modulator, the un-enhanced modulator (the optimal electrode structure from [5], but without the reactive terminations) and a commercial broadband modulator [7]. The measurement was made by connecting the ports of a vector network analyzer to the RF input port of the modulator and RF output port of the photodetector. Because each modulator has different optical insertion loss, all measurement data was normalized to give average optical power at the photodetector input of $0 \mathrm{dBm}$. The DC switching voltage of the commercial modulator is $4 \mathrm{~V}$.

It can be seen that with the termination attached the modulator has a resonant frequency of $1.76 \mathrm{GHz}$. At this

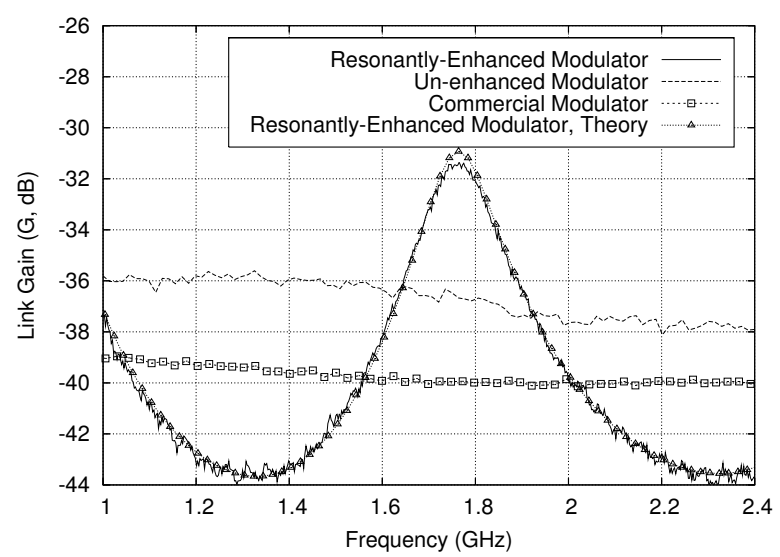

Fig. 2. Frequency response of the RF-photonic links.

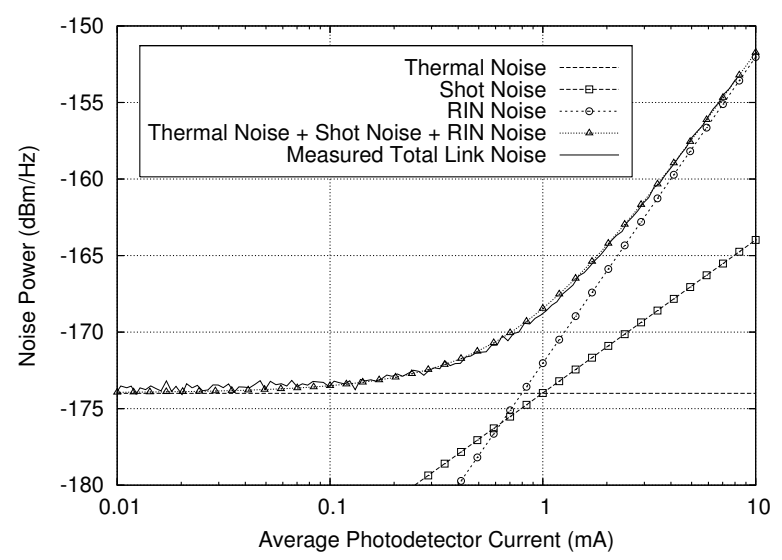

Fig. 3. Measured total link noise (at $1.76 \mathrm{GHz}$ ) and individual noise components, plotted as a function of average photodetector current.

resonant frequency, the resonantly-enhanced modulator exhibits greater than $5 \mathrm{~dB}$ improvement in link gain over the un-enhanced modulator. In comparing with the commercial modulator the resonantly-enhanced modulator provides approximately $9 \mathrm{~dB}$ link gain improvement. The $3 \mathrm{~dB}$ bandwith of the link using the resonantly-enhanced modulator is about $11 \%$. At the resonant frequency, the RF return loss $\left(S_{11}\right)$ of the resonantly-enhanced modulator is about $20 \mathrm{~dB}$.

The link gain performance of the resonantly-enhanced modulator calculated from the simulation model [8] is also presented in Fig. 2 for comparison with the measured data. It can be seen that the measured and simulated data have very good agreement.

To compensate for high optical insertion loss of the modulators, an erbium doped fiber amplifier (EDFA) with $10 \mathrm{~dB}$ gain was used. The relative intensity noise (RIN) of the laser and the EDFA was measured to be about $-153 \mathrm{~dB} / \mathrm{Hz}$. In Fig. 3, different noise components of the RF-photonic link were plotted against the average photodetector current. With the RIN level of $-153 \mathrm{~dB} / \mathrm{Hz}$, the link will be dominated by RIN noise when the photodetector current is above $2 \mathrm{~mA}$. Also shown in Fig. 3 is the measured RF-photonic link noise at different 


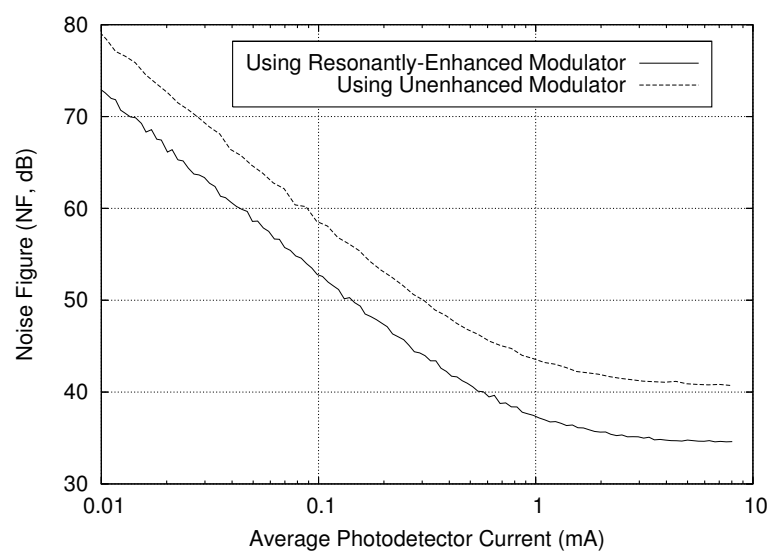

Fig. 4. Noise figure of the RF-photonic link using the resonantlyenhanced modulator in comparison with the link using the un-enhanced modulator at $1.76 \mathrm{GHz}$.

photodetector current levels. The total link noise was measured with an electrical spectrum analyzer and a low noise preamplifier. The RF input of the modulator was terminated with $50 \Omega$ resistance. The measurement equipment was calibrated with a noise source before the actual link noise measurement was made. As shown in Fig. 3, the measured link noise has similar response to the calculated noise.

The link gain at different photodetector current levels was then measured and the link noise figure was calculated by the following equation:

$$
N F=10 \log \left(\frac{N_{o}}{k T B G}\right)
$$

where $N_{o}$ is the total link noise, $k T=-174 \mathrm{dBm}$ is the input thermal noise of the link, $B$ is the equivalent noise bandwidth of the link, and $G$ is the link gain. The results of the link noise figure for the RF-photonic links using the resonantly-enhanced modulator and unenhanced modulator are plotted as functions of average photodetector current in Fig 4. As expected, when the optical power is not very high where the links are dominated by the shot noise and thermal noise, the link noise figures decrease with increasing optical power. However, when the links enter the RIN noise limited regime, further increase of optical power does not improve the link noise figure. This is because when the link is RIN noise limited, both the link noise and the link gain are proportional to the square of the photodetector current.

It is evident from Fig 4 that for any level of photodetector current, the link that employs the resonantly-enhanced modulator provides more than $5 \mathrm{~dB}$ lower in noise figure than the link using the un-enhanced modulator. It can be seen that to improve the link noise figure, one can increase the optical power when the link is thermal or shot noise limited. However, when the link is dominated by RIN noise, the link noise figure can only be improved by increasing the modulator efficiency.

The third-order intermodulation distortion of the links was measured by applying RF signals of equal amplitude

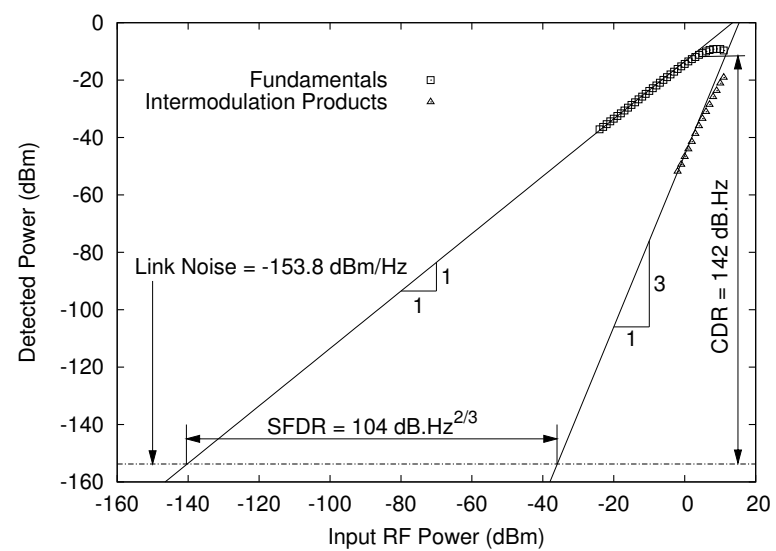

Fig. 5. Two-tone third-order intermodulation of the link using the resonantly-enhanced modulator with $7.7 \mathrm{~mA}$ average photodetector current.

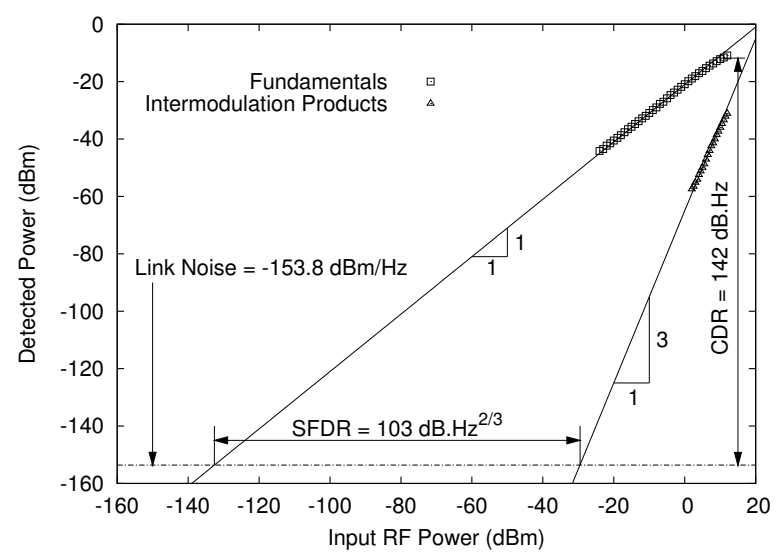

Fig. 6. Two-tone third-order intermodulation of the link using the un-enhanced modulator with $7.7 \mathrm{~mA}$ average photodetector current.

at close frequencies $f_{1}=1.755 \mathrm{Ghz}, f_{2}=1.765 \mathrm{GHz}$ and measuring the detected power at the fundamental $f_{1}, f_{2}$ and third-order intermodulation products $2 f_{1}-f_{2}, 2 f_{2}-$ $f_{1}$ frequencies with an electrical spectrum analyzer. The measured results are shown in Fig. 5 and Fig. 6 for the links using the resonantly-enhanced modulator and un-enhanced modulator respectively. Both links were operating in the RIN noise limited region with $7.7 \mathrm{~mA}$ of average photodetector current for minimum noise figures. At this level of photodetector current, the link noise was measured to be about $-153.8 \mathrm{dBm} / \mathrm{Hz}$. It can be seen that with RIN noise limited, both RF-photonic links exhibit the same spurious-free dynamic range (SFDR) and $1 \mathrm{~dB}$ compression dynamic range (CDR). Thus, the resonantlyenhanced modulator does not cause any improvement or degradation to the link distortion.

\section{Conclusions}

In this paper, we have theoretically and experimentally demonstrated an RF-photonic link using the resonantlyenhanced modulator at $1.8 \mathrm{GHz}$. At the resonant frequency, the resonantly-enhanced modulator offers more 
than $5 \mathrm{~dB}$ link gain improvement over the un-enhanced modulator. The same improvement factor in noise figure was obsevered when the RF-photonic link is RIN noise limited. The dynamic range of the link is not effected by the resonant enhancement. The design and realization of resonant modulators at higher frequency is currently under investigation.

\section{ACKNOWLEDGMENT}

The authors would like to thank the technical staff at the MMTC, School of Electrical and Computer Engineering, RMIT University for the fabrication of the devices.

\section{REFERENCES}

[1] A. Nirmalathas, D. Novak, C. Lim, R. Waterhouse, and D. Castleford, "Fiber networks for wireless applications," LEOS 2000. 2000 IEEE Annual Meeting Conference Proceedings. 13th Annual Meeting. IEEE Lasers and Electro-Optics Society 2000 Annual Meeting (Cat. No.00CH37080), pp. 35-36 Volume 1, 2000.

[2] A. Lindsay, G. Knight, and S. Winnall, "Photonic mixers for wide bandwidth rf receiver applications," IEEE Trans. Microwave Theory Tech., vol. 43, no. 9, pp. 2311-2317, 1995.

[3] T. Kawanishi, S. Oikawa, K. Higuma, Y. Matsuo, and M. Izutsu, "Resonant electrode structures for band-operation-type optical modulators," Proceedings of SPIE - The International Society for Optical Engineering, vol. 4870, pp. 577-586, 2002.

[4] R. Krahenbuhl and M. Howerton, "Investigations on short-pathlength high-speed optical modulators in $\mathrm{LiNbO}_{3}$ with resonanttype electrodes," Journal of Lightwave Technology, vol. 19, no. 9, pp. 1287-1297, 2001.

[5] T. G. Nguyen, A. Mitchell, and Y. S. Visagathilagar, "Investigation of resonantly enhanced modulators on $\mathrm{LiNbO}_{3}$ using FEM and numerical optimization technique," J. Lightwave Technol., vol. 22, no. 2, pp. 526-533, 2004.

[6] Y. Visagathilagar, A. Mitchell, and R. Waterhouse, "Fabry-perot type resonantly enhanced mach-zehnder modulator," in International Topical Meeting on Microwave Photonics. MWP'99. Technical Digest (Cat. No.99EX301), 1999, pp. 17-20 Volume 1.

[7] "Mach-10 external modulator," Covega Corporation.

[8] Y. Visagathilagar, A. Mitchell, and M. Austin, "General theoretical model for resonantly enhanced optical modulators," in Proc. 8th OptoElectronics Communication Conf. (OEC), vol. 23, 2003, pp. 337-338. 